\title{
DNN based Classification of ADHD fMRI Data using Functional Connectivity Coefficient
}

Nishant Chauhan and Byung-Jae Choi

Department of Electronic Engineering, Daegu University, Gyeongsan, Korea

\section{]jfis}

Received: Nov. 9, 2020

Revised : Dec. 4, 2020

Accepted: Dec. 4, 2020

Correspondence to: Byung-Jae Choi

(bjchoi@daegu.ac.kr)

@The Korean Institute of Intelligent Systems

(c) This is an Open Access article distributed under the terms of the Creative Commons Attribution Non-Commercial License (http://creativecommons.org/licenses/ by-nc/3.0// which permits unrestricted noncommercial use, distribution, and reproduction in any medium, provided the original work is properly cited.

\begin{abstract}
Functional magnetic resonance imaging (fMRI) has emerged as a popular research topic in neuroimaging for automated classification and recognition of different brain disorders. Attention Deficit Hyperactivity Disorder (ADHD) is one of the most common behavioral disorders in young children because its underlying mechanism is still not completely understood. The use of fMRI data in ADHD research is utilized to reflect the neural mechanism and functional integration of the brain. Alteration in the functional connectivity of the brain is expected to provide useful information for classifying or predicting brain disorders. In this study, a deep neural network (DNN) approach was applied to classify ADHD using functional connectivity-based fMRI data. The functional connectivity coefficient was extracted between regions determined by independent component analysis (ICA) and used to feed the DNN for classification. The DNN model demonstrated an accuracy of 95\% with the preprocessed fMRI data from Nilearn, which is a Python module for neuroimaging data.
\end{abstract}

Keywords: Functional Magnetic Resonance Imaging (fMRI), Deep Neural Network (DNN), Attention Deficit Hyperactivity Disorder (ADHD), Independent Component Analysis (ICA), Nilearn

\section{Introduction}

The advancement in methods for studying human brain structure and its functionality is one of the great landmarks of medical research [1]. The human brain can speculate a huge and complex network that efficiently controls the complicated systems of the human body. The brain regions continuously send and receive information to perform bodily functions. The temporal correlation of these regions is assumed to be functionally connected. Recently, we have seen a spike in brain imaging research for analysis of the brain mechanism and pathophysiology involved in neurodegenerative diseases and associated mental disorders. The analysis of functional connectivity of brain regions has become more popular because it is believed that functional connectivity can be key to understanding the cognitive processes of the brain [2].

Recent research has shown that some brain disorders, such as Parkinson's, Alzheimer's, and ADHD, are the outcome of the alteration of functional connectivity of the brain network [3]. To understand the mechanism of the disorder, it is important to precisely identify the altered functional connectivity. ADHD is the most common brain disorder found in young children and is characterized by hyperactivity, lack of attention, and behavior control issues [4]. Like many other brain disorders, ADHD increases the difficulty in life not only for the 
patient but also for a family [5]. Studies have shown that reduced or altered functional connectivity between the anterior cingulate cortex (ACC) and posterior cingulate cortex (PCC) is detected in the ADHD induced brain [6].

There are many imaging techniques available to study brain structure and physiology. Magnetic resonance imaging (MRI) provides structural information about the brain. Diffusion tensor imaging (DTI) displays the fiber connections between brain regions, and positron emission tomography (PET) captures the regional cerebral blood flow (CBF). To investigate the functional connectivity of the brain, functional magnetic resonance imaging (fMRI) has emerged as a favorable neuroimaging tool [2]. In particular, fMRI has the potential to identify the connectivity alteration caused by brain disorders such as Alzheimer's, epilepsy, schizophrenia, and ADHD.

In this paper, a DNN model is introduced to show the classification of ADHD using fMRI data based on functional connectivity analysis. This approach is not directly based on neuroimaging scans but rather on vectorized functional connectivity measures. The functional connectivity coefficients were extracted using independent component analysis (ICA) and used for training and testing for DNN.

The rest of the paper is organized into the following sections: Section 2 provides a brief literature review of existing methods. Section 3 explains the materials and methods used in the proposed classification model. Section 4 contains the results and discussion. Finally, the concluding remarks are provided in Section 5 .

\section{Literature Review}

Based on fMRI techniques, many researchers have investigated brain functionality and discovered the structural and functional abnormalities between individuals with ADHD and the control (healthy) group. Nakao et al. [7] used voxel-based morphological analysis in ADHD and found a decrease in the gray matter volume of the lenticular nucleus. A detection algorithm for ADHD using L2-regularized logistic regression and fMRI features was proposed and showed prominent results. However, for non-linear data, the detection accuracy decreased [8, 9]. A functional anatomical discriminative region model for pattern classification and discriminant feature identification was proposed in which ICA was used to extract functional networks in the brain [10]. ICA based methods are considered to be favorable for fMRI studies because there is no prior information required regarding spatial or temporal patterns of source signals. However, these methods have some limitations. First, it is difficult to understand the ICA components, and it is based on assumptions on the independence of components, either spatially or temporally. In addition, the selection of the threshold value for independent component maps and component counts might become uncertain and problematic [11].

In recent years, machine learning and deep learning have been applied to various study areas and shown incredible results. The analysis of fMRI data using machine learning and deep learning techniques is said to be the future of neuroimaging. A convolutional neural network approach was proposed for the recognition of Alzheimer's disease and showed high accuracy [12, 13].

Correlation is a well-known approach to estimate function connectivity, and there are 65 different correlation methods known to compute the functional connectivity of the brain. Dai et al. used segmentation to obtain 351 regions of interest (ROIs) of the brain and calculated the functional connectivity using Pearson's correlation [15].

In this study, a canonical ICA analysis was performed on fMRI data. Among these regions, functional connectivity coefficients have been extracted based on their connectivity parameters and used for classification using DNN.

\section{Materials and Methods}

We proposed a framework for the classification of individuals with ADHD and controls (healthy brain). Figure 1 illustrates the framework of this study. First, all preprocessed fMRI data were decomposed using ICA. It provided a unique representation of data and maps of activities that are as spatially independent

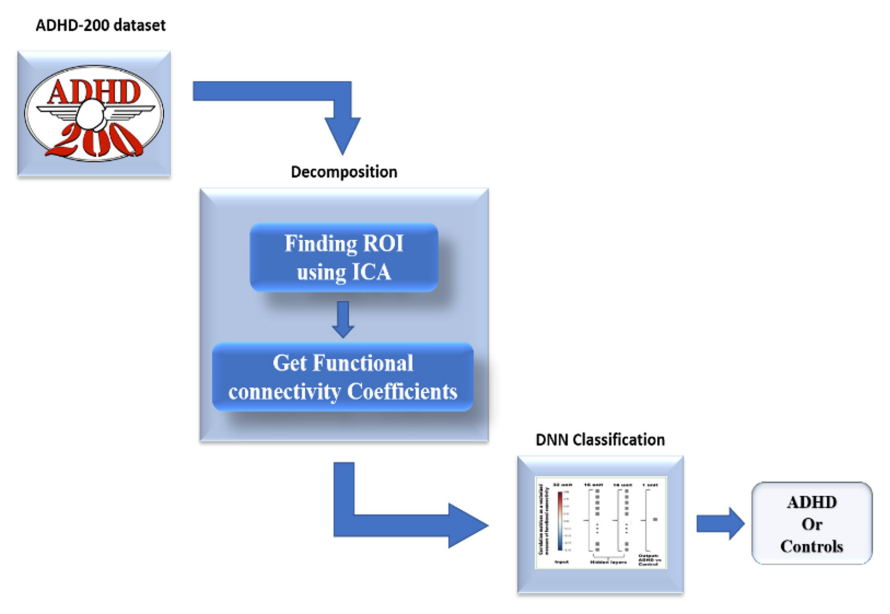

Figure 1. ADHD classification framework. 
as possible. Subsequently, functional connectivity coefficients were extracted and feature vectors of these coefficients used to train and test the DNN model for final classification. Furthermore, connectivity matrices for ADHD versus controls were also formed to visualize functional connectivity.

\subsection{Dataset}

In this study, we used ADHD-200 publicly accessible data composed of resting-state fMRI (rs-fMRI) and anatomical data accumulated from multiple research institutes [16]. This data was preprocessed by three teams using their preferred tools. The first team named their preprocessing the Athena pipeline, in which they used the tools from the FSL and AFNI packages; the second team named it NIAK pipeline, which is based on a neuroimaging analysis kit from CBRAIN software; and the third team carried out the voxel-based morphometry processing using the SPM8 tool, which is called the Burner pipeline. There were 40 data subjects' and four types of information: (1) paths to rs-fMRI data images (func), (2) CSV files that contain nuisance variables (confounds), (3) preprocessing step details (phenotypic), and (4) data description (description). The dataset featured both typically developing individuals called "controls" and those diagnosed with ADHD called "treatments."

\subsection{Decomposition}

Decomposition was the first step in extracting functional connectivity coefficients. ICA is commonly used to analyze fMRI data. To estimate functional connectivity, it is important to determine the appropriate ROIs. Nilearn has a function for group-level ICA called CanICA, which allows single subject variability control with respect to functional networks [17]. As recommended by Nilean documentation, 20 component decompositions have been chosen and independent components have been estimated. These independent components have been plotted on default anatomical brain images using a statistical atlas map to show the default mode network (DMN). The total components yielded by decomposition have been shown using a probabilistic atlas map on default anatomical brain images (Figure 2).

However, decomposition was still difficult to understand as we saw different brain regions. To resolve this issue, we summarized the brain signals obtained using ICA and transformed the extracted data into time series. Next, we determined the functional connectivity coefficient based on correlation, which is found to be more accurate in classification than other con-
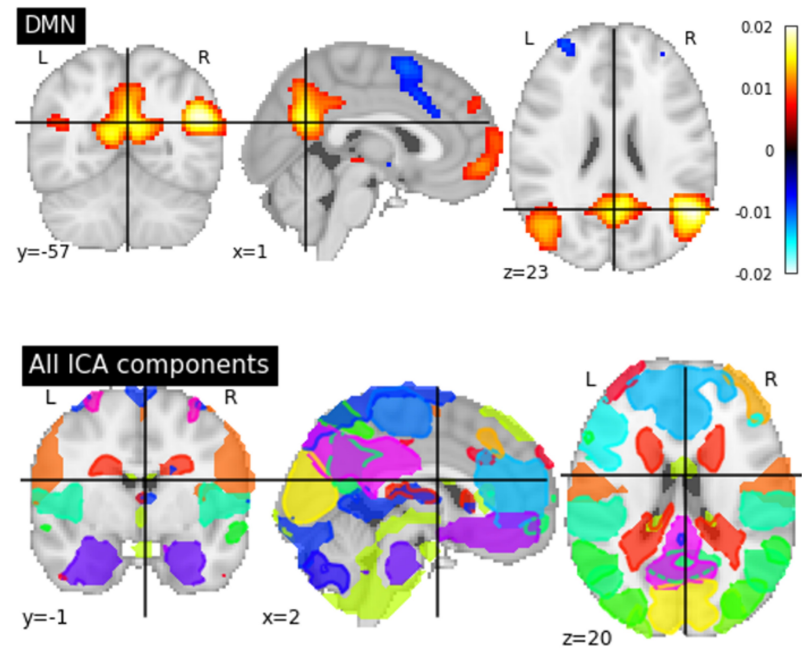

Figure 2. Decomposition using ICA.
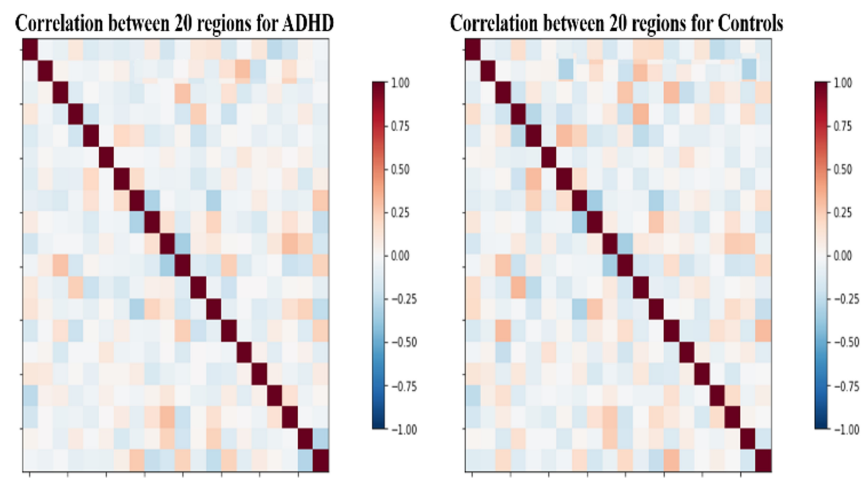

Figure 3. Functional connectivity coefficients matrices based on correlation.

nectivity parameters (tangent, partial correlation). Correlation simply calculates the marginal connectivity between pairwise ROIs. To compute correlation matrices, Nilearn provides a builtin function called ConnectivityMeasures, which requires two parameters: functional connectivity type (correlation) and time series data acquired from the previous step. This will calculate the connectivity matrices for all subjects. To check the average connectivity coefficient matrices, 20 ADHD (treatments) and 20 control subjects were chosen. Figure 3 shows the average connectivity matrices.

The diagonal lines in Figure 3 can be ignored, as they show correlation with themselves. For better visualization, we can plot the functional connectivity coefficients matrices on default brain image (Figure 4).

The ADHD connections seem less dense compared to the control ones, which indicated the reduced functional connec- 


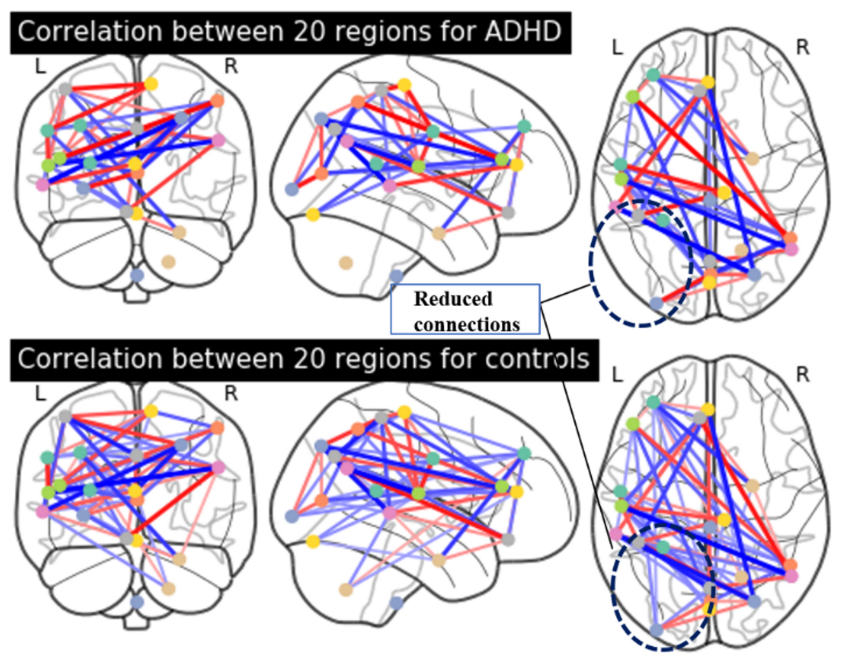

Figure 4. Functional connectivity coefficients visualization.

tivity caused by ADHD. The fewer connections in the superior parietal cortex (circled), which is responsible for attention, seemed altered due to ADHD (Figure 4). The collected correlation matrices that provide a vectorized measure of functional connectivity provided the input data for our DNN model for classification.

\subsection{Deep Neural Network Model}

A four-layer sequential model was used for classification with a specific number of nodes $(32,16,16,1)$. The output node was 1 because it is a binary classification. The reason for choosing four layers was because we were dealing with functions that were not necessarily linearly separable. The input length was set to 32 because the ADHD-200 data set had 40 subjects. The model architecture is shown in Figure 5.

\section{Results and Discussion}

The DNN classification models were implemented using the Intel PC (Intel Core i5-4590, 16 GB RAM, Nvidia GEFORCE GTX 1070 Ti GPU). Python 3.6 and TensorFlow 1.5 libraries with CUDA 10.0 enable were utilized to implement these models. Some Python libraries, such as Keras, Numpy, Pandas, Matplotlib, and Nilearn were used.

For activation functions, we used Tanh and Sigmoid activation for the first and last layer, respectively, because both are pointed by the vanishing gradient problem. In two hidden layers, the ReLu activation function was used to overcome the problem of hidden layers.

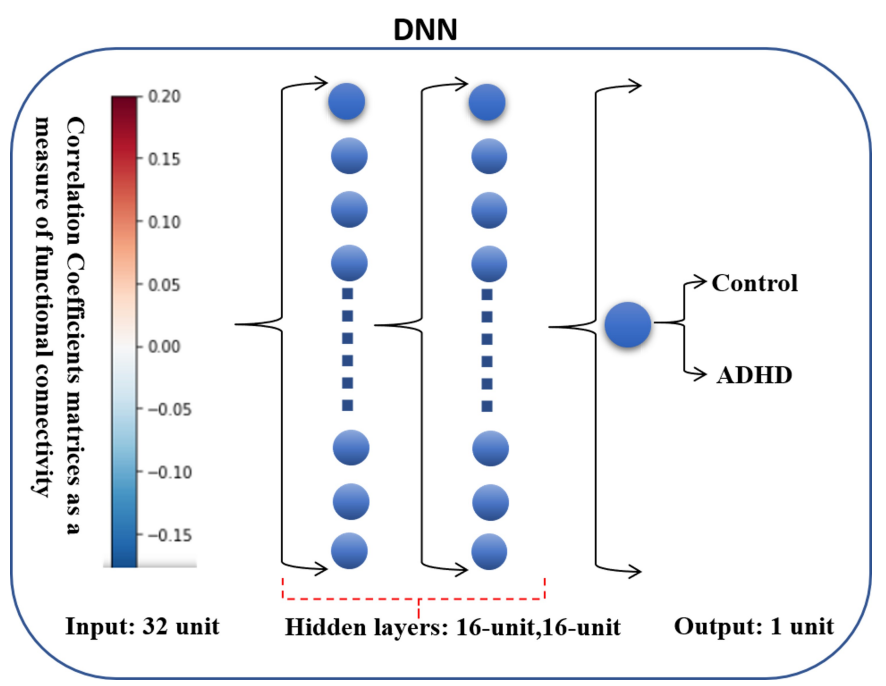

Figure 5. DNN model architecture.

Finally, Adam optimization and binary-cross entropy loss functions were used for training the DNN. The reason to select Adam was because it has shown good performance on noisy data. In addition, binary-cross entropy is a good choice for classification problems because it ensures that one output vector is unaffected by other factors, and it is independent for each class. The learning rate and epochs for the model were set to 0.0001 and 100, respectively, with a batch_size of 32 .

To check the performance of the DNN model, we used accuracy as an evaluation metric [18]. Accuracy(A) can be measured as the number of all correct predictions divided by the total number of predictions/datasets:

$$
A=\frac{T P+T N}{T P+T N+F N+F P} .
$$

The best accuracy value was $1.0(100 \%)$ and the worst was 0.0 $(0 \%)$.

Figure 6(a) shows the model accuracy graph. As shown in Figure 6, the training and testing accuracy increases with epochs and model loss and decreases with epochs. The DNN models achieved $95 \%$ accuracy, which showed that our decision to select correlation as a functional connectivity matrix was successful. These findings showed that classification models in fMRI are not always dependent on imaging data. Functional connectivity coefficients can be used for classification and detection in brain disorders, such as ADHD. 


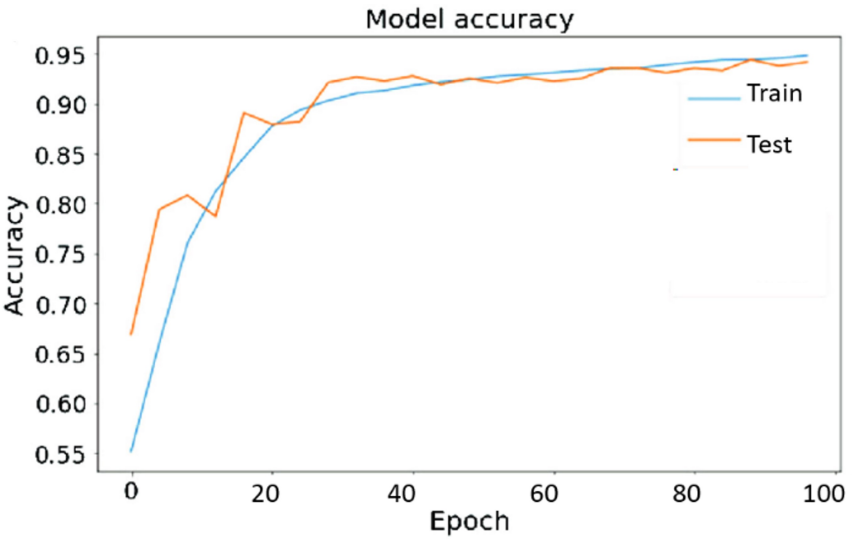

(a)

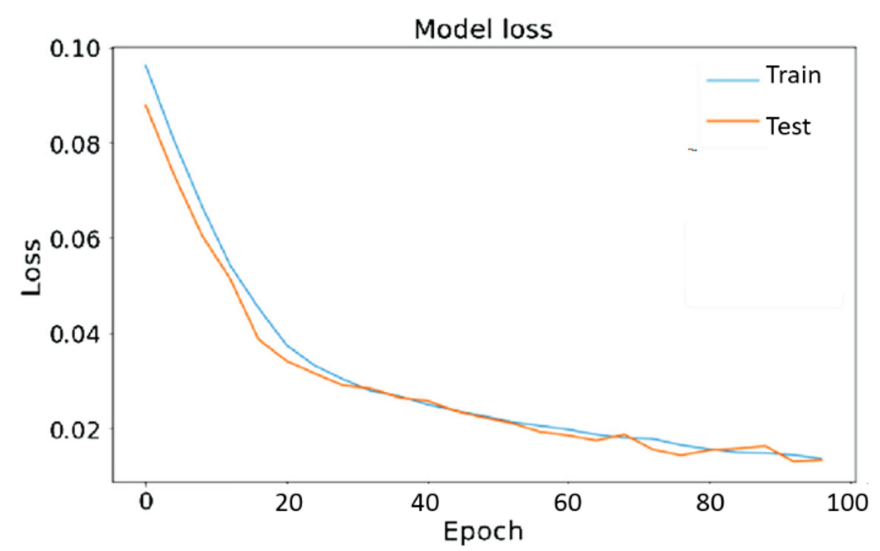

(b)

Figure 6. (a) DNN model accuracy and (b) DNN model loss.

\section{Concluding Remarks}

In this study, we explored how functional connectivity can be used to classify fMRI data for ADHD. Functional connectivity correlation coefficients for multiple ROIs were extracted using ICA, and the DNN model was used for classification. The model showed $95 \%$ classification accuracy. It will be interesting to see more work with a larger dataset and manual preprocessing steps using Nilearn built-in functions. The results of this study suggested that the DNN model has the potential to recognize individuals with ADHD.

\section{Acknowledgments}

This research was partly supported by the Daegu University and Korea Institute of Advancement of Technology (KAIT) grant funded by the Korean government (MOTIE) (P0012724, The Competency Development Program for Industry Specialist).

\section{Conflicts of Interest}

No potential conflict of interest relevant to this article was reported.

\section{References}

[1] N. Chauhan and B. J. Choi, "Performance analysis of classification techniques of human brain MRI images," International Journal of Fuzzy Logic and Intelligent Systems, vol. 19, no. 4, pp. 315-322, 2019. https://doi.org/10. 5391/ijfis.2019.19.4.315

[2] A. M. Bastos and J. M. Schoffelen, "A tutorial review of functional connectivity analysis methods and their interpretational pitfalls," Frontiers in Systems Neuroscience, vol. 9, article no. 175, 2016. https://doi.org/10.3389/fnsys. 2015.00175

[3] I. Singh, "Beyond polemics: science and ethics of ADHD," Nature Reviews Neuroscience, vol. 9, no. 12, pp. 957-964, 2008. https://doi.org/10.1038/nrn2514

[4] D. Tomasi and N. D. Volkow, "Abnormal functional connectivity in children with attention-deficit/hyperactivity disorder,' Biological Psychiatry, vol. 71, no. 5, pp. 443450, 2012. https://doi.org/10.1016/j.biopsych.2011.11.003

[5] G. V. Polanczyk, G. A. Salum, L. S. Sugaya, A. Caye, and L. A. Rohde, "Annual research review: a metaanalysis of the worldwide prevalence of mental disorders in children and adolescents," Journal of Child Psychology and Psychiatry, vol. 56, no. 3, pp. 345-365, 2015. https://doi.org/10.1111/jcpp.12381

[6] H. Yang, Q. Z. Wu, L. T. Guo, Q. Q. Li, X. Y. Long, X. Q. Huang, R. C. K. Chan, and Q. Y. Gong, “Abnormal spontaneous brain activity in medication-naive ADHD children: a resting state fMRI study," Neuroscience Letters, vol. 502, no. 2, pp. 89-93, 2011. https: //doi.org/10.1016/j.neulet.2011.07.028

[7] T. Nakao, J. Radua, K. Rubia, and D. Mataix-Cols, "Gray matter volume abnormalities in ADHD: voxel-based metaanalysis exploring the effects of age and stimulant medication," American Journal of Psychiatry, vol. 168, no. 11, pp. 1154-1163, 2011. https://doi.org/10.1176/appi.ajp. 2011.11020281 
[8] J. R. Sato, M. Q. Hoexter, A. Fujita, and L. A. Rohde, "Evaluation of pattern recognition and feature extraction methods in ADHD prediction," Frontiers in Systems Neuroscience, vol. 6, article no. 68, 2012. https: //doi.org/10.3389/fnsys.2012.00068

[9] M. Kim, "Semi-supervised recursive learning of discriminative mixture models for time-series classification," International Journal of Fuzzy Logic and Intelligent Systems, vol. 13, no. 3, pp. 186-199, 2013. https://doi.org/10.5391/ IJFIS.2013.13.3.186

[10] M. Nunez-Garcia, S. Simpraga, M. A. Jurado, M. Garolera, R. Pueyo, and L. Igual, "FADR: functionalanatomical discriminative regions for rest fMRI characterization," in Machine Learning in Medical Imaging. Cham, Switzerland: Springer, 2015, pp. 61-68. https: //doi.org/10.1007/978-3-319-24888-2_8

[11] H. Ghanadian, M. Ghodratigohar, and H. Al Osman, "A machine learning method to improve non-contact heart rate monitoring using an RGB camera," IEEE Access, vol. 6, pp. 57085-57094, 2018. https://doi.org/10.1109/ ACCESS.2018.2872756

[12] S. Sarraf and G. Tofighi, "Deep learning-based pipeline to recognize Alzheimer's disease using fMRI data," in Proceedings of 2016 Future Technologies Conference (FTC), San Francisco, CA, 2016, pp. 816-820. https: //doi.org/10.1109/FTC.2016.7821697

[13] R. Zafar, S. C. Dass, A. S. Malik, N. Kamel, M. J. U. Rehman, R. F. Ahmad, J. M. Abdullah, and F. Reza, "Prediction of human brain activity using likelihood ratio based score fusion," IEEE Access, vol. 5, pp. 13010-13019, 2017. https://doi.org/10.1109/ACCESS. 2017.2698068

[14] D. Dai, J. Wang, J. Hua, and H. He, "Classification of ADHD children through multimodal magnetic resonance imaging," Frontiers in Systems Neuroscience, vol. 6, article no. 63, 2012. https://doi.org/10.3389/fnsys.2012. 00063

[15] W. Kirch, "Pearson's correlation coefficient," in Encyclopedia of Public Health. Dordrecht, The Netherlands:
Springer, 2008, pp. 1090-1091. https://doi.org/10.1007/ 978-1-4020-5614-7_2569

[16] A. Abraham, F. Pedregosa, M. Eickenberg, P. Gervais, A. Mueller, J. Kossaifi, A. Gramfort, B. Thirion, and G. Varoquaux, "Machine learning for neuroimaging with scikit-learn," Frontiers in Neuroinformatics, vol. 8, article no. 14, 2014. https://doi.org/10.3389/fninf.2014.00014

[17] Nilearn: statistics for neuroimaging in Python, Available https://nilearn.github.io/\#

[18] M. H. Zweig and G. Campbell, "Receiver-operating characteristic (ROC) plots: a fundamental evaluation tool in clinical medicine," Clinical Chemistry, vol. 39, no. 4, pp. 561-577, 1993. https://doi.org/10.1093/clinchem/39.4.561

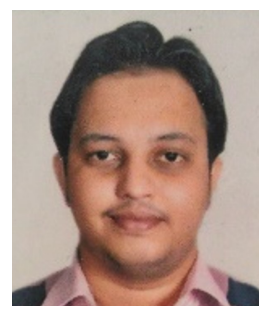

Nishant Chauhan received his B.S. degree in Computer Science from AKTU, India in 2012, and an M.S. degree in Control and Instrumentation from Daegu University, South Korea in 2020. He is currently a full-time Ph.D. student and working towards a Ph.D. degree in Control and Instrumentation at Daegu University, Department of Electronic Engineering (Graduate School). His research interests include intelligent control, fuzzy logic, Image processing, machine learning, and deep learning for medical images, Brain MRI, fMRI.

E-mail: nishantsep1090@daegu.ac.kr.

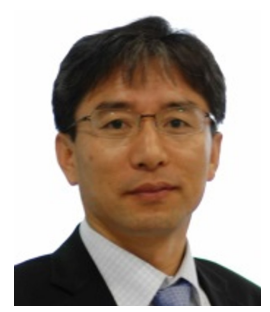

Byung-Jae Choi received his B.S. in Electronic Engineering, in 1987 from Kyungpook National University, Daegu. And he received his M.S. and a Ph.D. degree in Electrical and Electronic Engineering, 1989 and 1998, KAIST in Daejeon. He is a Professor of the School of Electronic and Electrical Engineering, Daegu University, Daegu, Korea, since 1999. His current research interests include intelligent control and its applications.

E-mail: bjchoi@daegu.ac.kr. 